\title{
A genome-wide cis-regulatory element discovery method based on promoter sequences and gene co-expression networks
}

\author{
Zhen Gao ${ }^{1 *}$, Ruizhe Zhao ${ }^{2}$, Jianhua Ruan ${ }^{\text {* }}$ \\ From The Eleventh Asia Pacific Bioinformatics Conference (APBC 2013) \\ Vancouver, Canada. 21-24 January 2013
}

\begin{abstract}
Background: Deciphering cis-regulatory networks has become an attractive yet challenging task. This paper presents a simple method for cis-regulatory network discovery which aims to avoid some of the common problems of previous approaches.
\end{abstract}

Results: Using promoter sequences and gene expression profiles as input, rather than clustering the genes by the expression data, our method utilizes co-expression neighborhood information for each individual gene, thereby overcoming the disadvantages of current clustering based models which may miss specific information for individual genes. In addition, rather than using a motif database as an input, it implements a simple motif count table for each enumerated $k$-mer for each gene promoter sequence. Thus, it can be used for species where previous knowledge of cis-regulatory motifs is unknown and has the potential to discover new transcription factor binding sites. Applications on Saccharomyces cerevisiae and Arabidopsis have shown that our method has a good prediction accuracy and outperforms a phylogenetic footprinting approach. Furthermore, the top ranked genemotif regulatory clusters are evidently functionally co-regulated, and the regulatory relationships between the motifs and the enriched biological functions can often be confirmed by literature.

Conclusions: Since this method is simple and gene-specific, it can be readily utilized for insufficiently studied species or flexibly used as an additional step or data source for previous transcription regulatory networks discovery models.

\section{Background}

The advance of experimental technology, including complete genome sequencing, high-throughput expression profiling [1-3] and binding-site mapping [4-6], has made the computational approach of studying the cis-regulatory networks (CRN) more attractive. A widely used model is to cluster genes based on their expression profile and then using motif finding algorithms [7-14] or motif enumerators to find the over-represented sequences within each cluster [15-18]. However, the correlation between gene clusters and motifs is imprecise because of the complex

\footnotetext{
* Correspondence: zgao@cs.utsa.edu; jruan@cs.utsa.edu 'Department of Computer Science, The University of Texas at San Antonio, One UTSA Circle, San Antonio, TX 78249, USA

Full list of author information is available at the end of the article
}

nature of regulation, as not all genes within a cluster share a common motif and the same motif can be found in gene promoters in other clusters. Importantly, these clustering based models are not suitable to model the expression of each individual gene. To overcome this limitation, [19] and [20] have proposed a linear regression model to predict statistically significant motifs. While a fascinating approach, it assumes that the number of occurrences of motifs in a promoter is linearly correlated with the gene expression, where motifs are identified by simply enumerating all $k$-mers. From an entirely different angle, [21] provided an approach that screened genomic sequences against a database of putative regulatory motifs, evaluating the contribution of the occurrences of the motif on the gene expression by comparing the expression profiles of 
genes containing this motif against those that do not. This method therefore requires knowledge about the putative motifs of that species, which may not always be available. Another major direction of motif finding and CRN discovery focuses on comparative genomics, also referred to as phylogenetic footprinting (PF) [22-34]. It assumes that the cis-regulation is conserved over evolution; thus, it does not need gene co-expression data as input to determine the sets of co-regulated genes. PF is a powerful method and has gained impressive success for the prediction of conserved regulatory elements. However, the main drawback of this method is that it cannot find species-specific regulatory elements.

Here, we propose a simple approach for inferring CRNs that avoids the common limitations mentioned above. Using genomic promoter sequences and gene expression data as input, our method utilizes the gene expression data in a novel way. In contrast to the approach favored by previous clustering based methods, which clusters genes by co-expression information and finds over-represented motifs for each cluster, our method first builds a gene co-expression network, and then searches for putative cis-regulatory elements that are enriched in the neighborhood for each individual gene on the network. In other words, our method utilizes the gene expression profiles in an individual gene motivated fashion. In addition, by using a simple enumerated $k$-mer counter to find the motif information within the promoter sequences, this approach needs little knowledge of the species-based putative motifs and requires few assumptions about the model by which elements of motif counts affect gene expression. It is a simple and versatile model for motif discovery and CRN finding. Thus, we hope it can be easily used for species with little previous cis-regulatory knowledge or be flexibly used for previous CRNs discovery approaches as an additional step. An in silico evaluation on Saccharomyces cerevisiae and Arabidopsis has been performed. Compared to a phylogenetic footprinting (PF) based method on several datasets, our method shows comparable or even better prediction accuracy. Furthermore, the top ranked cis-regulatory clusters uncovered by our approach for the two species are evidently functionally co-regulated, and the regulatory relationships between the motifs and the enriched biological functions can often be confirmed by literature.

\section{Methods}

\section{Cis-regulatory network construction}

The input of our method includes a list of promoter sequences and a gene-gene co-expression network (see Data sources). The $m$ promoter sequences are viewed as 'background' and the $n$ genes of the co-expression network are the target genes whose CRNs we want to study, where the $\mathrm{n}$ target genes are a subset of the $m$ background genes. The gene-gene co-expression network is represented by an adjacency matrix, $\mathbf{A}=\left\langle a_{i j}\right\rangle_{n \times n}$, where $a_{i j}=1$ if there is an edge between gene $i$ and gene $j$, and 0 otherwise. For convenience, we let $a_{i i}=1$ for all $i$. Here the number of target genes $n$ is from co-expression networks. The reason why we choose $n$ target genes out of $m$ is that in order to ensure a high-quality co-expression data, only the genes with the highest variations in the stress response data set will be chose to build co-expression network.

We first count the number of occurrences for each $k$-mer, where $k=6$ in this study, on each of the $m$ promoter sequences. Let $\mathbf{C}=\left\langle c_{i j}\right\rangle_{m \times l}$ be the $k$-mer occurrence table, where $l=4^{k}$ is the total number of candidate motifs $\left(k\right.$-mers). Let $\mathbf{D}=\left\langle d_{i j}\right\rangle_{n \times l}$ be a matrix derived from $\mathbf{C}$ such that each row of $\mathbf{D}$ corresponds to a row in $\mathbf{C}$ for the same gene's promoter, and the order of the genes in $\mathbf{D}$ is equivalent to that in $\mathbf{A}$.

Next, for each gene $g$ present in the co-expression network, we identify its neighbors in the network, $\pi_{g}=$ $\left\{i \mid a_{g i}=1\right\}$, and retrieve the corresponding rows from $\mathbf{D}$, defined as $\mathbf{T}^{g}=\left\langle t_{i j}\right\rangle=\left\langle d_{s j}\right\rangle$, where $s \in \pi_{g}$.

Finally, we compute the significance for the $j$-th $k$-mer being over-represented in the neighborhood of gene $g$ using either the cumulative hypergeometric test, or student's t-test. With the cumulative hypergeometric test, the $p$-value is calculated as

$$
p_{g j}=\sum_{r=x}^{\min (K, q)} \frac{\left(\begin{array}{l}
K \\
r
\end{array}\right)\left(\begin{array}{c}
m-K \\
q-r
\end{array}\right)}{\left(\begin{array}{c}
m \\
q
\end{array}\right)}
$$

where $x=\left|\left\{i \mid \mathbf{T}^{g}=\left\langle t_{i j}\right\rangle, t_{i j}>0\right\}\right|$ is the number of genes within gene $g$ 's neighborhood (including gene $g$ itself) that have at least one occurrence of motif $j, K=\left|\left\{i \mid c_{i j}>0\right\}\right|$ is the number of such genes in the background (whole genome), and $q=\left|\pi_{g}\right|$ is the number of neighbors for gene $g$ in the network. Intuitively, the cumulative hypergeometric $p$-value is the probability of drawing at least $x$ of a possible $K$ items in $q$ drawings without replacement from a group of $m$ objects.

On the other hand, with the student's t-test, the $p$-value is calculated by performing a non-paired two-sample t-test to compare the average occurrence of motif $j$ in the neighborhood of $g$ against that of the genes not in the neighborhood of $g$.

From the $p$-value matrix $\mathbf{P}=\left\langle p_{i j}\right\rangle_{n \times l}$, a score matrix $\mathbf{S}=\left\langle s_{i j}\right\rangle_{n \times l}$ can be computed by $s_{i j}=\log _{10} p_{i j}$. The values in $\mathbf{S}$ range from 0 to $+\infty$, where a greater number indicates a more significant regulatory relationship of a gene-motif pair. To cope with numerical precision limitations, all scores larger than 40 are converted to 40 . 
At last, the cis-regulatory network, represented by a bipartite graph $\mathbf{R}=(U, V, E, W)$ can be derived from $\mathbf{S}$, where $U$ is the set of genes, $V$ is the set of motifs, $E$ is the set of regulatory relationships, and $W$ is the associated edge weights, defined by $w_{i j}=s_{i j}$ for any edge. To ensure statistical significance and biological relevance, a cutoff is applied so that $\left(U_{i}, V_{j}\right) \in E$ if and only if $s_{i j}$ $>$ cutoff. In our study, for evaluation purposes, cutoff is set to 2 , corresponding to a $p$-value threshold at 0.01 . For visualization and biological analysis, cutoff is set to a much larger value (17 for yeast and 30 for Arabidopsis, see Results).

\section{Evaluation of the predicted cis-regulatory network}

To directly evaluate the predicted cis-regulatory network is difficult. We evaluated the performance of our algorithm indirectly in two ways.

\section{Co-regulatory network based evaluation}

First, following the idea in [34], we evaluate the CRNs based on co-regulatory networks. To this end, we first determine the similarity (Pearson correlation coefficient) between each pair of genes based on their motif scores. Subsequently, a co-regulatory network, $\mathbf{N}$, is constructed by connecting genes whose similarity scores are above a certain threshold. This co-regulatory network is then compared with some reference networks to determine the performance of our method. The key idea here is that if two genes share many regulatory elements, they are likely to be functionally related, and would share more edges with a reference network (see Data sources), which also captures functional relevance of genes. As the reference network and our predicted network may not have the same size, we first limit both networks to contain the same set of genes. We then compared the two networks using a set of standard metrics, defined as follows.

- True positive $(T P)$ is the number of edges in both $\mathbf{N}_{1}$ and $\mathbf{N}_{2}: T P=\left|Q_{1} \cap Q_{2}\right|$;

- False positive $(F P)$ is the number of edges in $\mathbf{N}_{1}$ but not $\mathbf{N}_{2}: F P=\mid Q_{1} \cap \overline{Q_{2}}$;

- True negative $(T N)$ is the number of non-edges in both $\mathbf{N}_{1}$ and $\mathbf{N}_{2}: T N=\left|\overline{Q_{1}} \cap \overline{Q_{2}}\right|$;

- False negative $(F N)$ is the number of edges in $\mathbf{N}_{2}$ but not $\mathbf{N}_{1}: F N=\left|Q_{2} \cap \overline{Q_{1}}\right|$;

- Precision, also referred to as the positive predictive value (PPV), is the ratio between TP and number of edges of $\mathbf{N}_{1}: P P V=\frac{T P}{\left|Q_{1}\right|}=\frac{T P}{T P+F P}$;

- Recall, also referred to as sensitivity or true positive rate (TPR), is the ratio between TP and number of edges of $\mathbf{N}_{2}: T P R=\frac{T P}{\left|Q_{2}\right|}=\frac{T P}{T P+F N}$.

Here $\mathbf{N}_{1}$ and $\mathbf{N}_{2}$ represent the predicted co-expression network and the reference co-expression network, respectively, $Q_{i}$ represent the set of edges in $\mathbf{N}_{i}$, and $\overline{Q_{i}}$ represents the set of edges in the inverse graph of $\mathbf{N}_{i}$.

\section{Model based evaluation}

Second, we evaluate the CRN using a completely different strategy. Our idea is that, if the CRN is correct and complete, we should be able to use it to model gene transcriptional level changes with a high accuracy. Therefore, given a set of gene expression microarray data, we attempt to construct a linear regression model for each microarray to predict the expression levels for each gene using the linear combination of the gene's motif scores. Importantly, in order to perform an unbiased evaluation, the gene expression microarray data used for evaluating the predicted cisregulatory network should be different from the gene expression data that we have used for constructing the gene co-expression network or cis-regulatory network (see Data sources).

Formally, let $e_{g}$ be the logarithm base two of the ratio of mRNA levels between two conditions for gene $g$, we model $e_{g}$ by $e_{g}=\sum_{i=1}^{k} \beta_{i} w_{i g}+c$. This formulation is identical to the popular model proposed by [19], except that they used motif occurrences (i.e., the $\mathbf{D}$ matrix), while we use the motif significance score (i.e., the $\mathbf{S}$ score and with some statistical cutoff). As the number of $k$-mer motifs (4096) we have is larger than the number of genes (3000), we apply a simple feature selection by only including the top $q(q$ «3000) motifs that have the highest correlation between motif significance scores and gene expression levels, and perform linear regression only using these top motifs. To measure the accuracy of the model, we calculated the root mean squared error (RMSE) of the linear model, as well as the Pearson correlation coefficient (PCC) between the predicted expression levels and the actual values. A higher PCC or a lower RMSE indicates a better prediction accuracy and therefore a biologically more relevant CRN.

\section{Competing methods}

We compare our method with two alternative methods. The first method is based on a naive model where we simply score each promoter sequence by counting the number of occurrences of each $k$-mer ( $k=6$ as in our main model). It is expected that such a simple method will su er from high false positive (i.e., a given $k$-mer may not be functional) and false negative (e.g., a motif instance may be missed due to mismatch). Nevertheless, this is the model used in most methods attempting to model gene transcriptional changes (e.g. $[19,20])$. The second method was proposed by [34], where they used phylogenetic footprinting to identify putative cis-regulatory elements in yeast Saccharomyces cerevisiae by discovering over-represented motifs in the promoters of their orthologs in 19 Saccharomycetes species. In their method, cis-regulatory elements are represented by dyads, i.e. pair of trinucleotides separated by a spacing comprised between 0 and $20 \mathrm{bp}$. 
The output of their method is similar to the gene-motif $p$ value matrix and we applied the same logarithm transformation as in our method so that a higher score means a more significant regulatory relationship.

\section{Data sources}

\section{Promoter sequences}

Promoter sequences for Saccharomyces cerevisiae (budding yeast) are downloaded from RSA tools [35]. The promoter sequence for each target gene is defined as 500 base pairs upstream from its transcription start site (TSS), or the whole intergenic region between the TSS and the coding sequence of the upstream gene, whichever is shorter.

Promoter sequences for Arabidopsis thaliana are downloaded from TAIR (arabidopsis.org). Promoter sequences are defined as 1000 base pairs upstream to the first annotated nucleotide of the gene (regardless of UTR or coding), according to TARI10 assembly.

\section{Microarray data and gene co-expression networks}

To construct a gene co-expression network, we used gene expression microarray data from [36], which contains the yeast gene expression data in response to a variety of environmental changes. This data set contains 173 arrays, and as in most previous studies, we selected the top 3000 genes with the highest variances [36]. After quantile normalizing the expression data, we constructed a co-expression network using the method described in [37]. Brie y, we first computed the Pearson correlation coefficient between the expression profiles of every pair of genes, and then ranked the correlation coefficients for each gene separately. Two genes are connected by an edge if the correlation between their expression profiles is ranked above a certain threshold within both genes' rankings. The threshold is determined automatically from an analysis of the resulting network's topological properties as described in [37]. The optimal rank threshold chosen is 120. Previous studies showed that such rank-based co-expression network can produce biologically more meaningful functional modules, especially for gene modules that are weakly co-expressed or conditionally co-expressed [37].

To evaluate the predicted cis-regulatory network for yeast, we used a separate yeast gene expression microarray data from [38], which contains 77 arrays measuring gene expression under various cell cycle phases.

Gene expression microarray data for Arabidopsis is obtained from AtGenExpress, which includes more than 1391 microarrays for various growth conditions, developmental stages, and tissues of Arabidopsis [39,40]. The gene co-expression network is constructed similarly as above. The optimal rank threshold chosen is 100 .

\section{Reference networks}

The choice of a proper reference network is crucial. In this work, we choose to evaluate our predicted CRN for Saccharomyces cerevisiae using two reference networks that are constructed also based on the principle of coregulation. The first reference network, the annotated regulon network, is constructed by linking pairs of genes belonging to the same annotated regulon that have been proved by low-throughput experiments $[41,42]$. The second reference network, known as the co-binding network, is derived from high-throughput ChIP-chip data [6], where we link any pair of genes that are shown to be bound by at least one common TF in the ChIP-chip data (with $p$-value $<0.001$ ). The annotated regulon network is smaller and less complete than the co-binding network, but probably more accurate. Table 1 shows some basic statistics of the networks. It is worth noting that the same networks were used by the competing method [34] in their paper to evaluate their co-regulatory networks.

\section{Results and discussion}

\section{The predicted cis-regulatory network (CRN) of} Saccharomyces cerevisiae

\section{The predicted co-regulatory network has a good accuracy}

First, as shown in Figure 1, the model that simply includes the promoter sequences information (denoted by 'cis, simple ct') has a very poor performance. The precision $(P P V)$ is almost always lower than $20 \%$. The red solid line shows the improvement by including both the promoter sequences and the neighborhood information from the coexpression networks, indicating that including the gene neighborhood information is critical for this method.

Compared to [34] using the annotated regulon network, our precision is higher when the recall is less than 0.04 or

Table 1 Size of the comparison networks

\begin{tabular}{lccccc}
\hline & \multicolumn{2}{c}{$\begin{array}{c}\text { Annotated co-regulation } \\
\text { network }\end{array}$} & \multicolumn{2}{c}{$\begin{array}{c}\text { Chip-chip co-binding } \\
\text { network }\end{array}$} & similarity matrix of ref [34] \\
\cline { 2 - 5 } & Nodes & Edges & Nodes & Edges & Nodes \\
\hline Original size & 612 & 10,599 & 2,397 & 178,202 & 3,146 \\
Similarity matrix of ref [34] & 446 & 5,816 & 1,491 & 77,597 & 3,146 \\
Cis-similarity matrix & 467 & 6,554 & 1,439 & 73,982 & 1,909 \\
Intersect with both & 360 & 3,984 & 1,006 & 39,693 & 1,909 \\
\hline
\end{tabular}

This table shows the size of the intersections of the networks that are restricted by the nodes (genes names) of the sub-networks. 'Cis-similarity matrix' represents our predicted co-regulatory similarity matrix. 


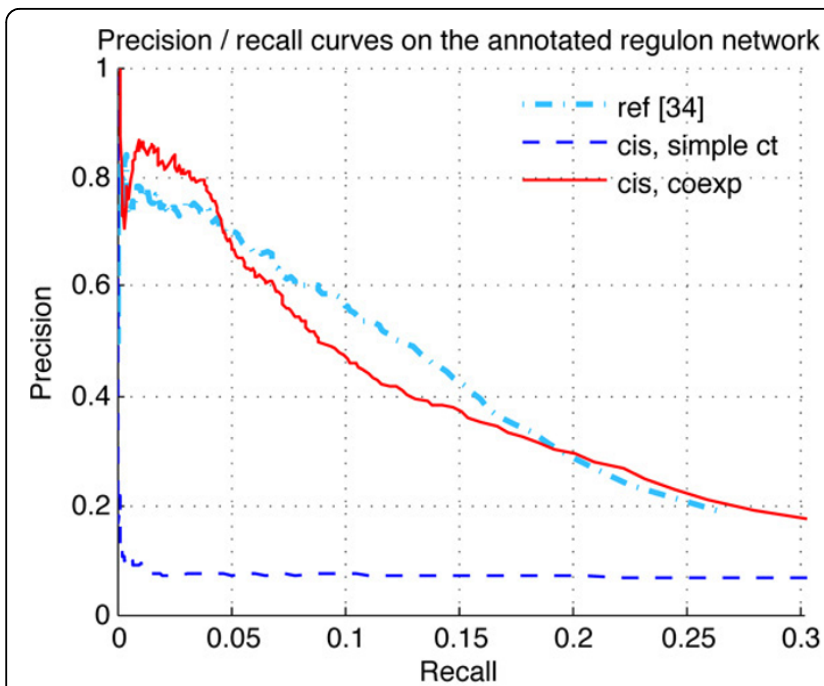

(a)

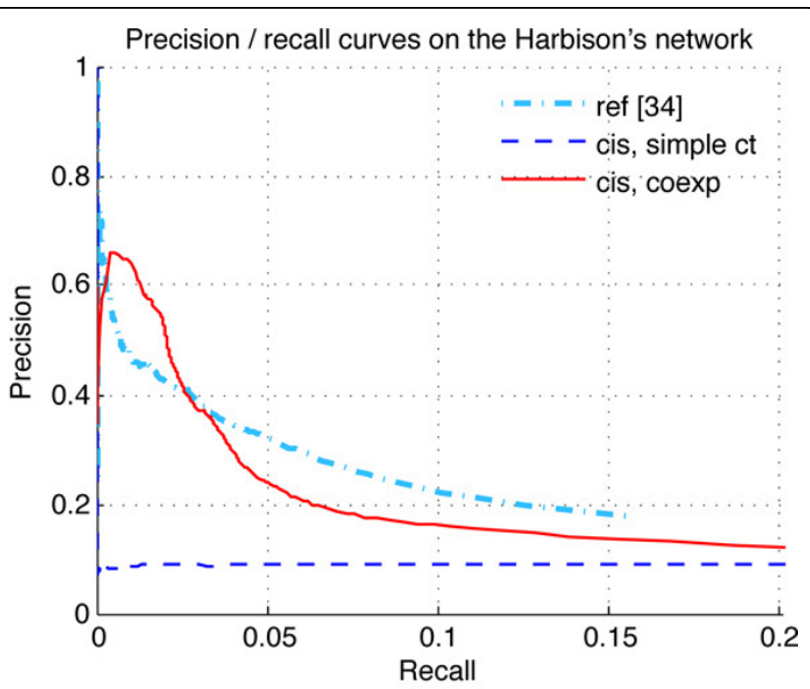

(b)

Figure 1 Co-regulatory network based evaluation results. Evaluation result of three models were shown - the reference model developed by Brohée, et al., 2011 (denoted by 'ref [34]'), the naive model with simple k-mer counts (denoted by 'cis, simple ct'), and our main model with both sequence information and gene expression neighborhood information from the gene co-expression network (denoted by 'cis, coexp'). (a) The comparison on the annotated regulon network. (b) The comparison on the Harbison's ChIP-chip based co-binding network.

greater than 0.2 (Figure 1a). The comparison using the ChIP-chip co-binding network [6] shows that, when the recall is less than 0.025 , our precision is better (Figure $1 b$ ). The advantage for the top predictions indicates that our method is better for species-specific predictions. These results show that the prediction accuracy of our approach is comparable with the phylogenetic footprinting approach for co-regulatory network prediction.

Since the predicted co-regulatory networks under comparison are intersected with annotated-regulon network or Chip-chip co-binding network, the evaluation covers only a small part of the original predicted co-regulatory networks, so the real prediction accuracy cannot be fully represented by these evaluated results. Compared to the annotated-regulon network, the $P P V$ of high-scoring prediction, with the score cutoff greater than 0.2 , is higher than $50 \%$. Thus, among the 90189 edges of the 3000 by 3000 original predicted co-regulatory network with cutoff $=0.2$, there should be a large portion of edges that correspond to real coregulations.

The predicted cis-regulatory network has a good correlation with the gene expression profiles

We evaluated the predicted cis-regulatory network by using it to model the gene expression level changes, and then finding the accuracy of the predicted expression level. It is important to note that the reference microarray gene expression dataset [38] used for modeling here is different from the gene expression dataset [36] used for constructing the co-expression networks and cisregulatory network. From Figure 2, it can be seen that our main model has the best result based on both the correlation and the root mean square error (RMSE). The result from the PF method ('Ref [34]') is significantly lower than our main method and only slightly better than the naive model. This is in sharp contrast to the evaluation results based on co-regulatory networks, where both methods significantly outperformed the naive method. One possible explanation is that while the PF method can predict well-conserved cis-regulatory elements with high accuracy, it will miss species-specific motifs, which are important to accurately model the gene expression levels. In addition, we attempted to predict the cell cycle gene expression data using the co-expression network (which was constructed from the stress-response data and a starting point of our algorithm) directly with the linear regression model. This result is shown by the fourth sets of bar in Figure 2. It shows that although the co-expression network performs the second best, it is still a little weak compared to our method.

Since our goal is to reveal the regulatory network, in other word, to find the relationship between the transcription factors and the target genes, the overall good quality of modeling gene expression data doesn't tell the best part of our approach. If we can find examples that the input gene expression profile cannot modeling the evaluating gene expression profile well, but our genemotif score matrix can, that would show the value of our method. Thus, we provided the figure in additional file 1 . The $\mathrm{x}$ axis shows the 77 gene expression conditions of 


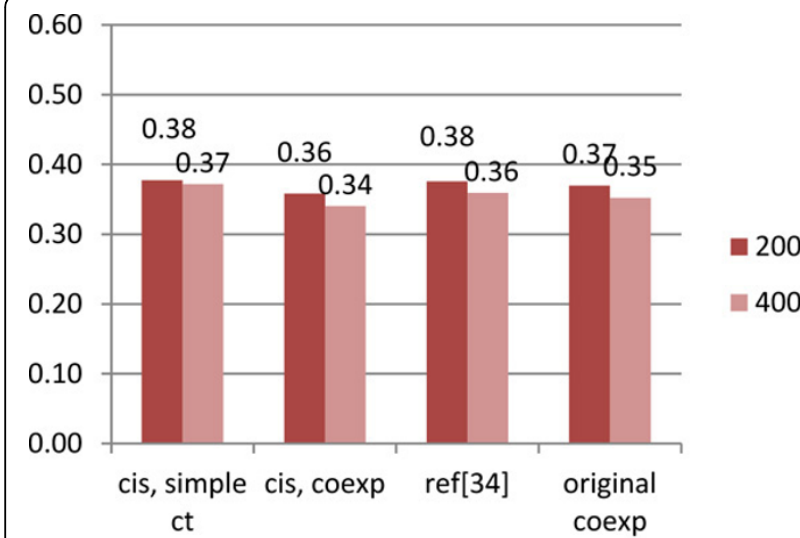

(a)

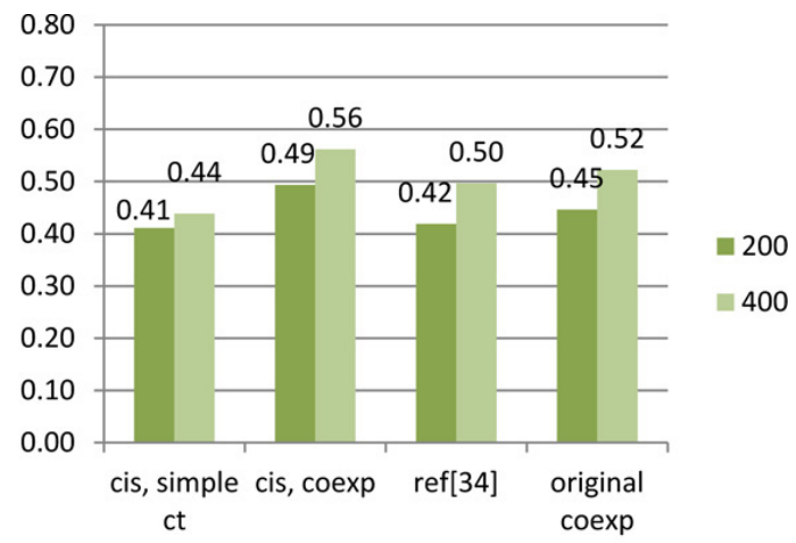

(b)

Figure 2 Linear regression evaluation result. It shows the RMSE (a) and the correlation (b) between the prediction values of the linear models (which are based on the cis-regulatory matrices) and the gene expression profiles. The three cis-regulatory element finding approaches are the naive model with simple $k$-mer counts (denoted by 'cis, simple $c t^{\prime}$ ), our main model with both sequence information and gene expression neighborhood information (denoted by 'cis, coexp'), and the reference model developed by Brohée, et al., 2011 (denoted by 'ref [34]') The fourth column shows the modeling result for the gene expressions by the original input co-expression network of our method. The legend on the right of each bar chart indicates the number of top k-mers used to build the linear model and predict the expression level (see Methods). A lower RMSE or a higher correlation indicates a better prediction accuracy.

the evaluating gene expression profile. The y axis shows the correlation between the predicted expression level and the true expression level. The red curve shows the results of our approach and the blue curve shows the results of the original input co-expression network of our approach. Both of the prediction results are using the top 400 features. From the figure, we can see that for some of the conditions, our model performs better than the original input co-expression profile. In addition, the modeling factors of our approach are the k-mers which uncover the potential TF binding sites.

\section{The predicted cis-regulatory network for yeast is biologically relevant}

We have performed a preliminary analysis to evaluate the biological significance of the predicted cis-regulatory networks. For efficiency, we only focused on the most significant portion of the network. Using a cutoff 17 on the the motif significance score matrix, $\mathbf{S}$ (see Methods), we produced a sparse CRN that contains several relatively large and dense clusters. Figure 3 and 4 shows the overall network topology and the six selected clusters, respectively.

Then, we analyzed the functions of genes in the discovered cis-regulatory clusters using the DAVID Functional Annotation Clustering tool. Table 2 shows the discovered cis-regulatory elements and annotated functional clusters for the six cis-regulatory modules shown in Figure 4. The significance of the functional annotations is measured by the Fisher's exact test for the enrichment level of the function within the cluster relative to the genome-wide genes with this function [43]. As shown, all six clusters are significantly enriched with some functional terms. More importantly, literature search confirmed that the cis-regulatory elements identified in each module matched the binding sites of transcription factors that are known to regulate the corresponding biological process. For example, the consensus motif regulating the subnetwork in Figure 4a, GATGAGC, resembles the wellknown PAC motif (GATGAG), which has been shown recently to be the binding sites of two transcription factors Pbf1 and Pbf2 and regulates ribosome biogenesis [44]. The module in Figure $4 \mathrm{~b}$ contains multiple cis-regulatory elements that appear to be subsequences of the Rap1-binding motif, CACCCRWACA [45], which is known to be present in most yeast ribosomal protein genes [46]. The cis-regulatory elements in Figure 4c include the known binding sites for Mbp1 (ACGCGT) and Swi4 (CGCGAA), two key regulators of yeast cell cycle [46]. The GATAAG motif in Figure $4 \mathrm{~d}$ matches the binding sites of several GATA-family TFs, including Gat1, Gln3, Dal80, and Gzf3, all of which are known to be involved in nitrogen metabolism [46]. Figure 4e contains the well-known stressresponse element (STRE, AAGGGG) bound by Msn2 and Msn4 proteins [45]. Finally, the GCACCC motif shown in Figure 4f matches perfectly with the binding sites of Aft1, which is known to be involved in iron utilization and homeostasis [46]. Therefore, the discovered cis-regulatory modules are functionally relevant. 


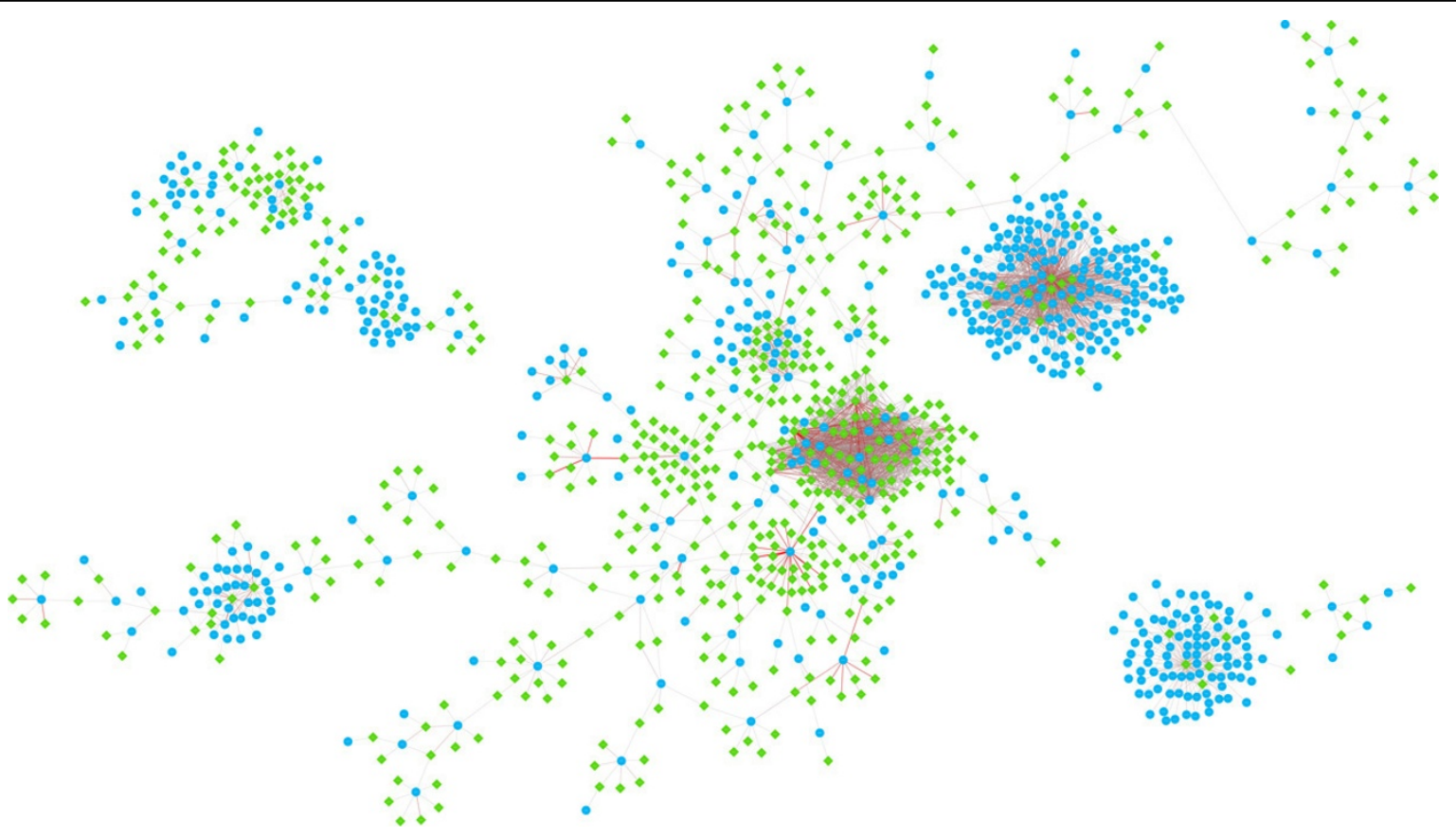

Figure 3 A predicted Saccharomyces cerevisiae cis-regulatory network. It is a whole cis-regulatory network of Saccharomyces cerevisiae predicted by this paper. Starting from the gene vs. motif significance score matrix, $\mathbf{S}$, we use a score cutoff of 17 to produce the cis-regulatory network, $\mathbf{R}$. The green diamonds represent 6-mer motifs and the blue ellipses represent genes. The color and width of the edges show the significance score. The color ranges from light gray to red (while the width ranges from 1 to 7), representing the significance score range from 17 to 126, respectively. The major clusters shown in this figure may have important biological meaning (see Figure 2 and Table 2).

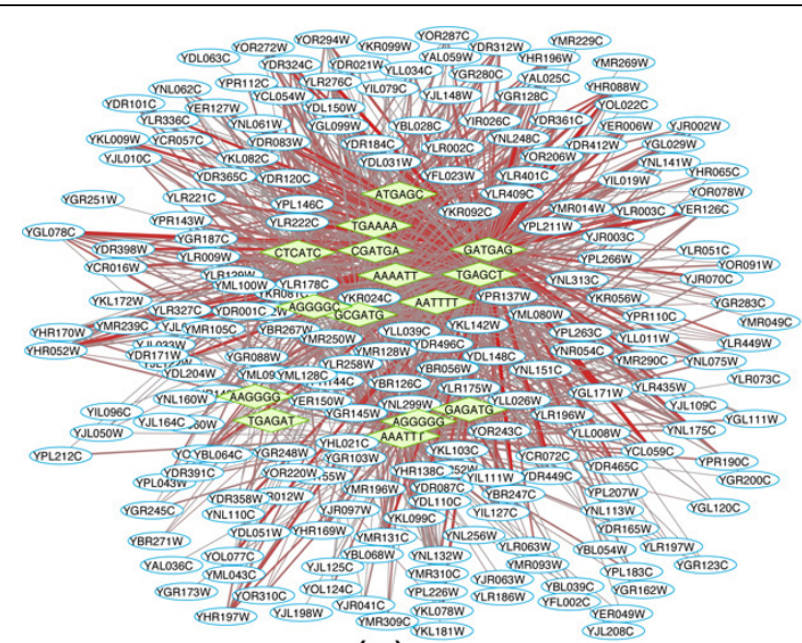

(a)

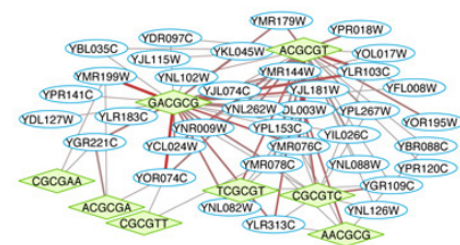

(c)

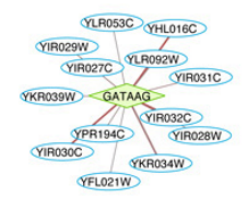

(d)

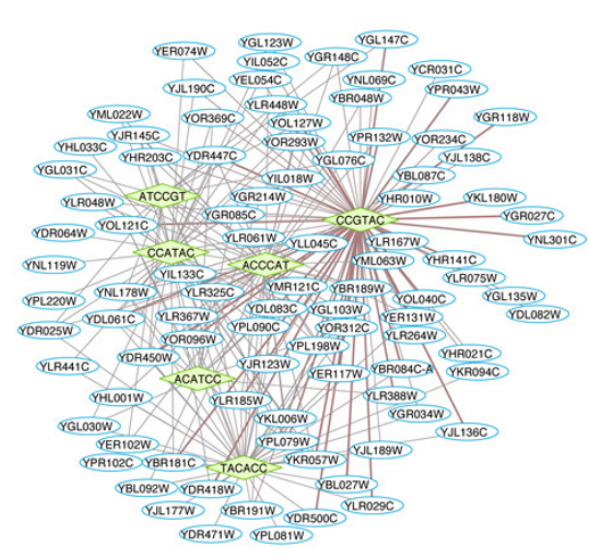

(b)

Figure 4 Six representative predicted clusters of Saccharomyces cerevisiae. The green diamonds represent 6-mer motifs and the blue ellipses represent genes. The graph visual style is changed from Fig. 3 in order to show the gene names clear. The functional clusters discovered by the DAVID Functional Annotation Clustering tool within the six cluster are shown by Table 2. 
Table 2 Discovered cis-regulatory clusters for Saccharomyces cerevisiae

\begin{tabular}{|c|c|c|c|c|c|}
\hline d-clust & d-count & related 6-mers & Functional Cluster Name & f-count & P-val \\
\hline \multirow[t]{5}{*}{ Fig. $4 a$} & 182 & $\begin{array}{l}\text { AAAATT, AAATT, AATTT, ATGAGC, CGATGA, } \\
\text { CTCATC, GAGATG, GATGAG, GCGATG, TGAAAA, } \\
\text { TGAGAT, TGAGCT, AAGGGG, AGGGGC, AGGGGG }\end{array}$ & nucleolus & 120 & $2.90 \mathrm{E}-129$ \\
\hline & & & ribosome biogenesis & 90 & $2.30 \mathrm{E}-108$ \\
\hline & & & ncRNA processing & 111 & $1.70 \mathrm{E}-94$ \\
\hline & & & 905 preribosome & 45 & $4.50 \mathrm{E}-47$ \\
\hline & & & nuclear lumen & 127 & $1.20 \mathrm{E}-97$ \\
\hline \multirow[t]{4}{*}{ Fig. $4 b$} & 85 & $\begin{array}{l}\text { TACACC, CCGTAC, CCATAC, } \\
\text { ATCCGT, ACCCAT, ACATCC }\end{array}$ & cytosolic ribosome & 82 & $9.70 \mathrm{E}-131$ \\
\hline & & & ribosome & 79 & $2.90 \mathrm{E}-128$ \\
\hline & & & structural constituent of ribosome & 82 & $6.40 \mathrm{E}-111$ \\
\hline & & & cytosolic small ribosomal subunit & 36 & $1.30 \mathrm{E}-51$ \\
\hline \multirow[t]{4}{*}{ Fig. $4 c$} & 36 & $\begin{array}{l}\text { AACGCG, ACGCGA, ACGCGT, } \\
\text { CGCGAA, CGCGTC, CGCGTI, } \\
\text { GACGCG, TCGCGT }\end{array}$ & chromosome & 16 & $6.00 \mathrm{E}-11$ \\
\hline & & & cell cycle & 23 & $9.00 \mathrm{E}-13$ \\
\hline & & & mitotic sister chromatid cohesion & 9 & $9.40 \mathrm{E}-12$ \\
\hline & & & DNA metabolic process & 19 & $6.60 \mathrm{E}-11$ \\
\hline \multirow[t]{2}{*}{ Fig. 4d } & 13 & GATAAG & amide catabolic process & 6 & $6.20 \mathrm{E}-13$ \\
\hline & & & purine metabolism & 5 & $3.40 \mathrm{E}-11$ \\
\hline \multirow[t]{3}{*}{ Fig. $4 \mathrm{e}$} & 30 & AAGGGG, AGGGGC, AGGGGG, & response to temperature stimulus & 21 & $6.30 \mathrm{E}-25$ \\
\hline & & & response to abiotic stimulus & 21 & $8.10 \mathrm{E}-21$ \\
\hline & & & vacuolar protein catabolic process & 14 & $4.20 \mathrm{E}-16$ \\
\hline \multirow[t]{3}{*}{ Fig. $4 f$} & 7 & GCACCC, GGGTGC & iron transport & 5 & $3.10 \mathrm{E}-09$ \\
\hline & & & iron ion transport & 5 & $2.60 \mathrm{E}-08$ \\
\hline & & & siderophore transport & 4 & 8.70E-08 \\
\hline
\end{tabular}

'd-clust' is the identifier for the discovered clusters; 'd-count' is the number of genes in the discovered cluster; 'f-count' is the number of genes in the functional clusters, which was discovered by previous well-organized experiments; 'P-value' is measured by Fisher's exact test p-value, which measures the enrichment level of the functions within the discovered cis-regulatory cluster relative to the genome.

\section{The predicted cis-regulatory network of Arabidopsis}

Similar to the process of evaluating the predicted cisregulatory network for Saccharomyces cerevisiae, using an $S$ cutoff of 30, we determine the cis-regulatory network for Arabidopsis (Figure 5). For the six most interesting clusters (Figure 6), we present the discovered annotated functional clusters in Table 3 from the DAVID Functional Annotation Clustering tool. The Arabidopsis is much more poorly annotated compared to yeast, and much less is known about the cis-regulatory networks in Arabidopsis. Nevertheless, the cis-regulatory modules identified by our method still shows significant functional coherence. For example, several motifs in Figure $6 \mathrm{~b}$ match to the core subsequence (CACGTG) of the well-known abscisic acid responsive element (ABRE) [47]. The CATGCA motif in Figure $6 \mathrm{c}$ is part of the RY-repeat element that is specifically required during seed development [47]. The motif GGCCCA in Figure $6 \mathrm{~d}$ matches the UP1ATMSD motif that is over-represented in a list of up-regulated genes after main stem decapitation in Arabidopsis, many of which are predicted to function in protein synthesis [48]. Finally, genes in Figure 6e are involved in cell cycle and are regulated by the CAACGG motif, which contains the myb core motif found in the promoter of Arabidopsis cell cycle regulating cyclin B1:1 gene [47].

\section{Conclusions}

The method provided by this paper combined gene expression profiles and promoter sequences in a novel way. By including the neighborhood information from the gene expression profiles rather than clustering the genes, it does not neglect the information of each individual gene in the expression profiles. The accuracy of the predicted co-regulatory network was high when compared to the annotated regulon network, the ChIP-chip co-binding network, and outperformed a phylogenetic footprinting based method [34]. Additionally, by using the motif enumerator, it is more flexible for discovering cis-regulatory elements in various species, or for improving current CRN discovering methods. Compared to current methods using phylogenetic footprinting, this method is better for discovering species specific coregulations. 


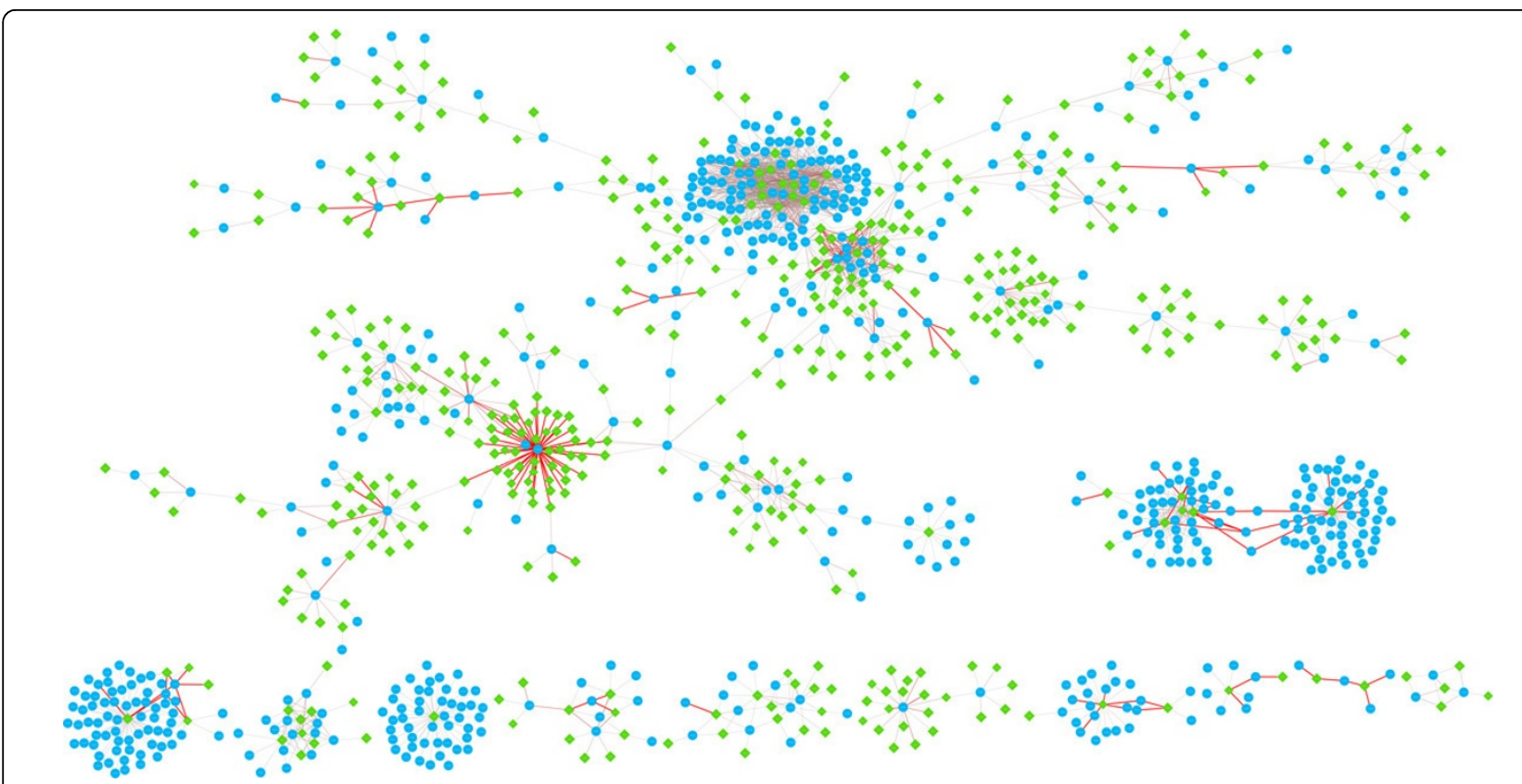

Figure 5 A predicted Arabidopsis cis-regulatory network. It is the graphical representation of the predicted cis-regulatory network, $\mathbf{R}$, for Arabidopsis, with cutoff 30 on the score matrix, $\mathbf{S}$. The color and the width of the edges indicate the significance score.

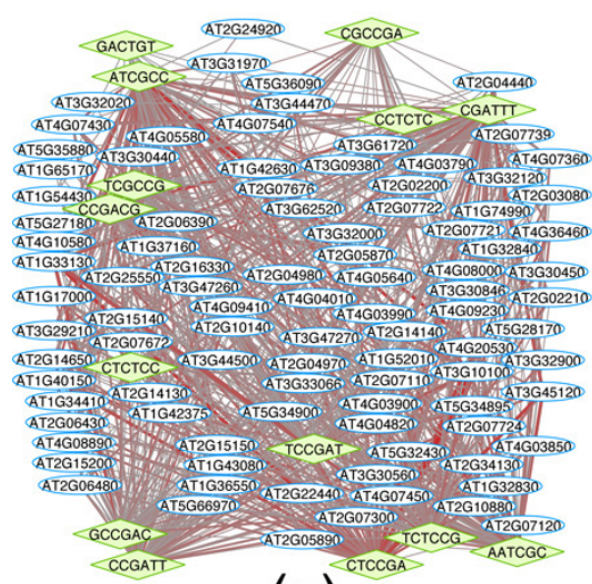

(a)

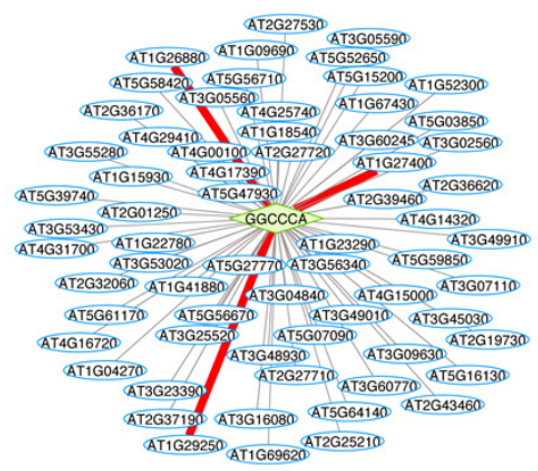

(d)

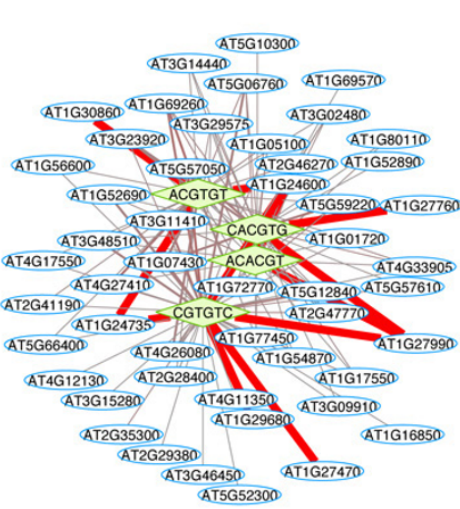

(b)

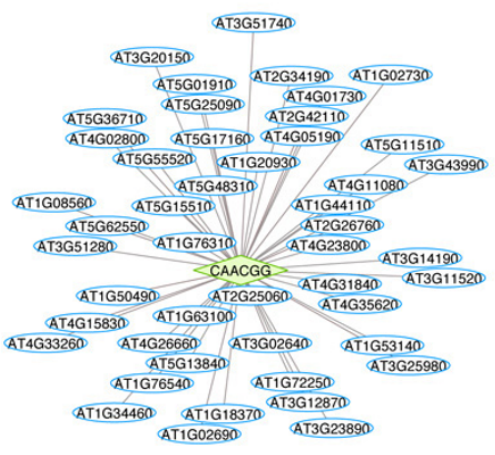

(e)

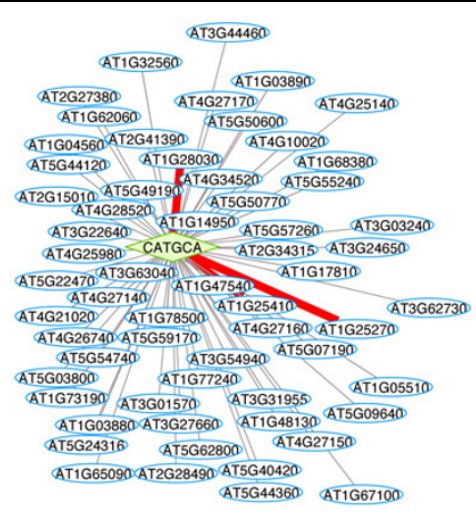

(c)

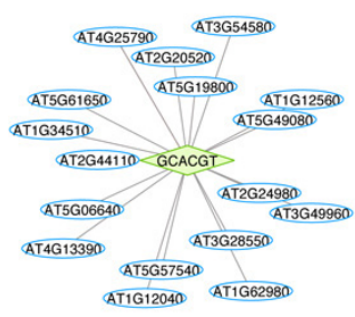

(f)

Figure 6 Six representative predicted clusters of Arabidopsis. Refer to Table 3 for the discovered functional clusters within the six predicted cis-regulatory clusters. 
Table 3 Discovered cis-regulatory clusters for Arabidopsis

\begin{tabular}{|c|c|c|c|c|c|}
\hline d-clust & d-count & related 6-mers & Functional Cluster Name & f-count & P-val \\
\hline Fig. 6a & 95 & $\begin{array}{l}\text { AATCGC, ATCGCC, CCGACG, CCGATT, CCTCTC, CGATTT, } \\
\text { CGCCGA, CTCCGA, CTCTCC, GACTGT, GCCGAC, TCCGAT, } \\
\text { TCGCCG, TCTCCG }\end{array}$ & cysteine-type peptidase activity & 8 & 4.60E-11 \\
\hline \multirow[t]{4}{*}{ Fig. 6b } & 50 & ACACGT, ACGTGT, CACGTG, CGTGTC & response to water deprivation & 11 & $1.50 \mathrm{E}-11$ \\
\hline & & & response to abscisic acid stimulus & 12 & 7.90E-11 \\
\hline & & & PP2C SIG & 8 & $9.80 \mathrm{E}-11$ \\
\hline & & & Protein phosphatase $2 \mathrm{C}$ & 8 & $1.20 \mathrm{E}-10$ \\
\hline \multirow[t]{2}{*}{ Fig. $6 c$} & 61 & CATGCA & nutrient reservoir activity & 12 & $1.80 \mathrm{E}-18$ \\
\hline & & & Seed storage protein & 7 & $6.50 \mathrm{E}-16$ \\
\hline \multirow[t]{4}{*}{ Fig. 6d } & 66 & GGCCCA & cytosolic ribosome & 64 & 1.90E-103 \\
\hline & & & cytosolic part & 61 & $3.10 \mathrm{E}-99$ \\
\hline & & & ribosomal protein & 65 & $3.00 \mathrm{E}-121$ \\
\hline & & & structural constituent of ribosome & 65 & 8.10E-104 \\
\hline \multirow[t]{2}{*}{ Fig. 6e } & 47 & CAACGG & cell cycle & 11 & $7.60 \mathrm{E}-13$ \\
\hline & & & cell division & 10 & $2.00 \mathrm{E}-12$ \\
\hline \multirow[t]{3}{*}{ Fig. $6 f$} & 17 & GCACGT & plant-type cell wall organization & 8 & $9.80 \mathrm{E}-14$ \\
\hline & & & Extensin-like region & 6 & 7.00E-13 \\
\hline & & & structural constituent of cell wall & 6 & 4.40E-11 \\
\hline
\end{tabular}

'd-clust' is the identifier for the discovered clusters; 'd-count' is the number of genes in the discovered cluster; 'f-count' is the number of genes in the functional clusters, which was discovered by previous well-organized experiments; 'P-value' is measured by Fisher's exact test $p$-value, which measures the enrichment level of the functions within the discovered cis-regulatory cluster relative to the genome.

The future work of this paper includes two directions. First, more advanced motif models could be used for improving the accuracy of regulatory network discovery. Currently, we just use the simplest approach in each step to show that the co-expression network-based approach can improve the specificity of cis-regulatory networks. For the 6-mer model, it may have more false positives compared with longer k-mer models. Later, we would like to try some state of the art approaches to identify cis-regulatory element. Second, better methods can be designed to identify genes that are not necessarily direct neighbors of a target gene, but are likely involved in similar biological processes. Finally, more tests could be done by applying this method on other species.

\section{Additional material}

\section{Additional file 1: The comparison of prediction accuracy for gene} expression between methods.

\section{Abbreviations}

CRN: cis-regulatory network; PF: phylogenetic footprinting; PCC: Pearson correlation coefficient: RMSE: root mean squared error; TSS: transcription start site.

\section{Acknowledgements}

This work was supported by NSF grants IOS-0848135, IIS-1218201, NIH grants SC3GM086305, U54CA113001, G12MD007591 (Computational Systems Biology (Core), and a UTSA Tenure-track Research Award.
Author details

'Department of Computer Science, The University of Texas at San Antonio, One UTSA Circle, San Antonio, TX 78249, USA. ${ }^{2}$ Ronald Reagan High School, 19000 Ronald Reagan, San Antonio, TX 78258, USA.

\section{Authors' contributions}

ZG designed the study, performed the data analysis and drafted the manuscript. RZ assisted in the data analysis and writing. JR conceived of the study, participated in its design and coordination and helped to draft the manuscript. All authors read and approved the final manuscript.

\section{Declarations}

The publication costs for this article were funded by $\mathrm{NIH}$ grant SC3GM086305.

This article has been published as part of BMC Genomics Volume 14 Supplement 1, 2013: Selected articles from the Eleventh Asia Pacific Bioinformatics Conference (APBC 2013): Genomics. The full contents of the supplement are available online at http://www.biomedcentral.com/ bmcgenomics/supplements/14/S1.

\section{Competing interests}

The authors declare that they have no competing interests.

Published: 21 January 2013

\section{References}

1. Fodor S, Rava R, Huang X, Pease A, Holmes C, Adams C: Multiplexed biochemical assays with biological chips. Nature 1993, 364:555-556.

2. Schena M, Shalon D, Davis R, Brown P: Quantitative monitoring of gene expression patterns with a complementary DNA microarray. Science 1995, 270(5235):467-470.

3. Tomancak P, Beaton A, Weiszmann R, Kwan E, Shu S, Lewis S, Richards S, Ashburner $M$, Hartenstein V, Celniker S, et al: Systematic determination of patterns of gene expression during Drosophila embryogenesis. Genome Biol 2002, 3(12):RESEARCH0088.

4. Ren B, Robert F, Wyrick J, Aparicio O, Jennings E, Simon I, Zeitlinger J, Schreiber J, Hannett N, Kanin E, et al: Genome-wide location and function of DNA binding proteins. Science's STKE 2000, 290(5500):2306. 
5. Iyer V, Horak C, Scafe C, Botstein D, Snyder M, Brown P: Genomic binding sites of the yeast cell-cycle transcription factors SBF and MBF. Nature 2001, 409(6819):533-538.

6. Harbison C, Gordon D, Lee T, Rinaldi N, Macisaac K, Danford T, Hannett N, Tagne J, Reynolds D, Yoo J, et al: Transcriptional regulatory code of a eukaryotic genome. Nature 2004, 431(7004):99-104.

7. Roth F, Hughes J, Estep P, Church G: Finding DNA regulatory motifs within unaligned noncoding sequences clustered by whole-genome mRNA quantitation. Nature biotechnology 1998, 16(10):939-945.

8. Hertz G, Stormo G: Identifying DNA and protein patterns with statistically significant alignments of multiple sequences. Bioinformatics 1999, 15(7):563-577.

9. Bailey T, Elkan C, University of California SDDoCS, Engineering: Fitting a Mixture Model by Expectation Maximization to Discover Motifs in Bipolymers Citeseer; 1994

10. Lawrence C, Altschul S, Boguski M, Liu J, Neuwald A, Wootton J: Detecting subtle sequence signals: a Gibbs sampling strategy for multiple alignment. Science 1993, 262(5131):208-214.

11. Workman C, Stormo G: ANN-Spec: a method for discovering transcription factor binding sites with improved specificity. Pac Symp Biocomput 2000, 467-478.

12. Buhler J, Tompa M: Finding motifs using random projections. Journal of computational biology 2002, 9(2):225-242.

13. Liu X, Brutlag D, Liu J: An algorithm for finding protein-DNA binding sites with applications to chromatin-immunoprecipitation microarray experiments. Nature biotechnology 2002, 20(8):835-839.

14. Sinha S, Tompa M: YMF: a program for discovery of novel transcription factor binding sites by statistical overrepresentation. Nucleic acids research 2003, 31(13):3586-3588.

15. van Helden J, André B, Collado-Vides J: Extracting regulatory sites from the upstream region of yeast genes by computational analysis of oligonucleotide frequencies. Journal of molecular biology 1998, 281(5):827-842.

16. Brāzma A, Jonassen I, Vilo J, Ukkonen E: Predicting gene regulatory elements in silico on a genomic scale. Genome research 1998, 8(11):1202-1215.

17. Beer M, Tavazoie S: Predicting gene expression from sequence. Cell 2004, 117(2):185-198.

18. Elemento O, Slonim N, Tavazoie S: A universal framework for regulatory element discovery across all genomes and data types. Molecular cell 2007, 28(2):337-350

19. Bussemaker H, Li H, Siggia E, et al: Regulatory element detection using correlation with expression. Nature genetics 2001, 27(2):167-171.

20. Foat B, Houshmandi S, Olivas W, Bussemaker H: Profiling conditionspecific, genome-wide regulation of mRNA stability in yeast. Proceedings of the National Academy of Sciences of the United States of America 2005, 102(49):17675

21. Pilpel $Y$, Sudarsanam $P$, Church G: Identifying regulatory networks by combinatorial analysis of promoter elements. Nature genetics 2001, 29(2):153-159.

22. Kellis $\mathrm{M}$, Patterson $\mathrm{N}$, Endrizzi $\mathrm{M}$, Birren $\mathrm{B}$, Lander $\mathrm{E}$, et al: Sequencing and comparison of yeast species to identify genes and regulatory elements. Nature 2003, 423(6937):241-254

23. Cliften P, Sudarsanam P, Desikan A, Fulton L, Fulton B, Majors J, Waterston R, Cohen $B$, Johnston M: Finding functional features in Saccharomyces genomes by phylogenetic footprinting. Science 2003, 301(5629):71-76.

24. Pritsker M, Liu Y, Beer M, Tavazoie S: Whole-genome discovery of transcription factor binding sites by network-level conservation. Genome research 2004, 14:99-108.

25. Wang T, Stormo G: Combining phylogenetic data with co-regulated genes to identify regulatory motifs. Bioinformatics 2003, 19(18):2369-2380.

26. Blanchette $M$, Tompa M: Discovery of regulatory elements by a computational method for phylogenetic footprinting. Genome research 2002, 12(5):739-748.

27. Lenhard B, Sandelin A, Mendoza L, Engström P, Jareborg N, Wasserman W: Identification of conserved regulatory elements by comparative genome analysis. Journal of Biology 2003, 2(2):13.

28. Krivan W, Wasserman W: A predictive model for regulatory sequences directing liver-specific transcription. Genome Research 2001, 11(9):1559-1566.

29. Oeltjen J, Malley T, Muzny D, Miller W, Gibbs R, Belmont J: Large-scale comparative sequence analysis of the human and murine Bruton's tyrosine kinase loci reveals conserved regulatory domains. Genome Research 1997, 7(4):315-329.
30. Hardison R: Conserved noncoding sequences are reliable guides to regulatory elements. Trends in Genetics 2000, 16(9):369-372.

31. Loots G, Locksley R, Blankespoor C, Wang Z, Miller W, Rubin E, Frazer K: Identification of a coordinate regulator of interleukins 4,13 , and 5 by cross-species sequence comparisons. Science 2000, 288(5463):136-140.

32. Wasserman W, Palumbo M, Thompson W, Fickett J, Lawrence C, et al: Human-mouse genome comparisons to locate regulatory sites. Nature genetics 2000, 26(2):225-228.

33. Qiu P, Qin L, Sorrentino R, Greene J, Wang L: Comparative promoter analysis and its application in analysis of PTH-regulated gene expression. Journal of molecular biology 2003, 326(5):1327-1336.

34. Brohée $S$, Janky R, Abdel-Sater $F$, Vanderstocken $G$, André $B$, van Helden J: Unraveling networks of co-regulated genes on the sole basis of genome sequences. Nucleic acids research 2011, 39(15):6340-6358.

35. Thomas-Chollier M, Defrance M, Medina-Rivera A, Sand O, Herrmann C, Thiery D, van Helden J: RSAT 2011: regulatory sequence analysis tools. Nucleic Acids Res 2011, 39:W86-W91.

36. Gasch A, Spellman P, Kao C, Carmel-Harel O, Eisen M, Storz G, Botstein D, Brown P: Genomic expressionprograms in the response of yeast cells to environmental changes. Mol Biol Cell 2000, 11:4241-4257.

37. Ruan J, Perez J, Hernandez B, Sunter G, Sponsel V: Systematic construction and analysis of co-expression networks for identification of functional modules and cis-regulatory elements. 9th International Workshop on Data Mining in Bioinformatics, in conjuction with SIGKDD'10 Washington DC, USA: ACM; 2010.

38. Spellman $P$, Sherlock $G$, Zhang $M$, lyer $V$, Anders $K$, Eisen $M$, Brown $P$, Botstein D, Futcher B: Comprehensive identification of cell cycleregulated genes of the yeast Saccharomyces cerevisiae by microarray hybridization. Molecular biology of the cell 1998, 9(12):3273-3297.

39. Schmid M, Davison TS, Henz SR, Pape UJ, Demar M, Vingron M, Schölkopf B, Weigel D, Lohmann J: A gene expression map of Arabidopsis development. Nat Genet 2005, 37:501-506.

40. Kilian J, Whitehead D, Horak J, Wanke D, Weinl S, Batistic O, D'Angelo, Bornberg-Bauer E, Kudla J, Harter K: The AtGenExpress global stress expression data set: protocols, evaluation and model data analysis of UV-B light, drought and cold stress responses. Plant J 2007, 50:347-363.

41. Wingender E, Dietze $P$, Karas H, Knüppel R: TRANSFAC: a database on transcription factors and theirDNA binding sites. Nucleic acids research 1996, 24:238-241.

42. Simonis N, Wodak S, Cohen G, Van Helden J: Combining pattern discovery and discriminant analysis to predict gene co-regulation. Bioinformatics 2004, 20(15):2370-2379.

43. Hosack D, Dennis G Jr, Sherman B, Lane H, Lempicki R, et al: Identifying biological themes within lists of genes with EASE. Genome Biol 2003, 4(10):R70.

44. Zhu C, Byers KJRP, McCord RP, Shi Z, Berger MF, Newburger DE, Saulrieta K, Smith Z, Shah MV, Radhakrishnan M, Philippakis AA, Hu Y, De Masi F, Pacek M, Rolfs A, Murthy T, LaBaer J, Bulyk ML: High-resolution DNAbinding specificity analysis of yeast transcription factors. Genome Research 2009, 19(4):556-566.

45. Badis G, Chan ET, van Bakel H, Pena-Castillo L, Tillo D, Tsui K, Carlson CD, Gossett AJ, Hasino MJ, Warren CL, Gebbia M, Talukder S, Yang A, Mnaimneh S, Terterov D, Coburn D, Yeo AL, Yeo ZX, Clarke ND, Lieb JD, Ansari AZ, Nislow C, Hughes TR: A library of yeast transcription factor motifs reveals a widespread function for Rsc3 in targeting nucleosome exclusion at promoters. Molecular Cell 2008, 32(6):878-887.

46. Dwight S, Balakrishnan R, Christie K, Costanzo M, Dolinski K, Engel S, Feierbach B, Fisk D, Hirschman J, Hong E, Issel-Tarver L, Nash R, Sethuraman A, Starr B, Theesfeld C, Andrada R, Binkley G, Dong Q, Lane C, Schroeder M, Weng S, Botstein D, Cherry J: Saccharomyces genome database: underlying principlesand organisation. Brief Bioinform 2004, 5:9-22.

47. Higo K, Ugawa Y, Iwamoto M, Korenaga T: Plant cis-acting regulatory DNA elements (PLACE) database:1999. Nucleic Acids Res 1999, 27:297-300.

48. Tatematsu K, Ward S, Leyser O, Kamiya Y, Nambara E: Identification of ciselements that regulate gene expression during initiation of axillary bud outgrowth in Arabidopsis. Plant Physiol 2005, 138(2):757-766.

doi:10.1186/1471-2164-14-S1-S4

Cite this article as: Gao et al:: A genome-wide cis-regulatory element discovery method based on promoter sequences and gene co-expression networks. BMC Genomics 2013 14(Suppl 1):S4. 